\title{
Modelo de garantía de calidad en educación virtual para pueblos indígenas
}

\author{
Tovar Caro, Edmundo, PhD. ${ }^{l}$ y Dolz, Fátima Consuelo, PhD. ${ }^{2}$ \\ ${ }^{1}$ Universidad Politécnica de Madrid, España, etovar@fi.upm.es; ${ }^{2}$ Universidad Mayor de San Andrés, \\ Boliva,fdolz@umsa.bo
}

\begin{abstract}
Resumen-En el trabajo de investigación que abordamos en este artículo, se ha construido y propuesto un modelo de garantía de calidad en educación no formal en modalidad virtual para pueblos indígenas del departamento de La Paz, Bolivia. Se ha ejecutado una serie de actividades, trabajos y proyectos aplicando y evaluando empíricamente el modelo en poblaciones indígenas. Posteriormente se ha realizado una evaluación formal con los datos empíricos obtenidos en dos proyectos de capacitación a poblaciones indígenas de la Amazonía boliviana, con lo cual se tiene el modelo de garantía de calidad validado habiéndose ajustado algunos elementos. En este documento se presenta el modelo de garantía de calidad en educación virtual para pueblos indígenas validado, para ser difundido y aplicado en Programas o Cursos de Educación Virtual dirigidos a poblaciones indígenas del departamento de La Paz.
\end{abstract}

Keywords-- Garantía de calidad, educación virtual, pueblos indígenas, modelo validado, educacion no formal.

\section{INTRODUCCIÓN}

Clasificados como campesinos por los respectivos gobiernos de los países en que habitan, la gran mayoría de los Pueblos indígenas vive en una pobreza extrema en las remotas áreas rurales donde a duras penas consiguen sobrevivir de la agricultura. Los campesinos indígenas constituyen el $60 \%$ de la población total de Bolivia [1]. Siendo la agricultura su principal actividad, resulta ddifícil conciliar actividades de educación regular con actividades rurales, habiendo también dificultad en el transporte estudiantil, y por la cantidad que representan, resulta difícil impartir educación Alternativa presencial para todas y cada una de las comunidades indígenas. Estos son problemas que afectan actualmente y han afectado mucho a generaciones anteriores, entonces es necesario estandarizar contenidos para masificar la educación rompiendo barreras de tiempo y espacio accesibles para estos pueblos. El acceso a TIC no es un fin por sí mismo sino que su uso debe atender los sectores menos favorecidos como la educación de pueblos indígenas, por tanto la propuesta es que los niños, jóvenes y principalmente adultos indígenas puedan acceder a una capacitación continua más amplia y flexible sin barreras de tiempo y espacio, y que se desarrolle en un marco de calidad educativa asegurando procesos enseñanza - aprendizaje que respondan a una educación y sociedad globalizada, y que permita al poblador indígena prepararse para desenvolverse en su ámbito social mediante aprendizajes significativos que le identifiquen, y afiancen su cultura ancestral. El trabajo de investigación al que nos referimos en este documento [2], en base a la problemática educativa detectada en poblaciones

Digital Object Identifier (DOI):

http://dx.doi.org/10.18687/LACCEI2016.1.1.357

ISBN: 978-0-9822896-9-3

ISSN: 2414-6390 indígenas de Bolivia y a fin de contribuir a mejorar la calidad de vida de poblaciones rurales indígenas posibilitando un manejo comprensivo de la tecnología, integrándola a las propuestas de educación alternativa continua así como planes de desarrollo, tanto en la vida cotidiana como en las actividades laborales y que responda a estándares de calidad ha orientado su objeto de estudio a la construcción, propuesta y validación de un modelo de garantía de calidad en Educación Alternativa no presencial para Pueblos Indígenas. En ese sentido se ha presentado la propuesta de Modelo teórico de Garantía de Calidad en Educación Virtual para Pueblos Indígenas, que se ha representado como un modelo causal en que tenemos variables de Entrada o variables exógenas, variables de Proceso o intermedias, y variables de Resultado o Finales, estableciéndose un orden de precedencia en que las variables de entrada o exógenas se preparan antes de iniciar cualquier programa de capacitación dirigido a Pueblos indígenas, y dan lugar al Proceso Enseñanza Aprendizaje (PEA) en modalidad virtual, así como a las relaciones que se establecen entre alumnos y profesores, y las interacciones entre la organización institucional y la Comunidad, que marca la diferencia siendo un modelo basado en la organización comunal indígena $\boldsymbol{A y l l u}$ [3].

\section{RE-ESPECIFICACIÓN DEL MODELO VALIDADO}

Luego de haber procedido con todos los pasos de la metodología causal [4]: formulación teórica del modelo, especificación, identificación, estimación y evaluación a lo largo del desarrollo de la investigación, habiendo validado el modelo construido, utilizando los datos empíricos obtenidos, se realiza ahora la re-especificación del modelo terminado y validado para su difusión y aplicación [5] [6]. Este modelo supone la esquematización de la realidad, constituido por aquellas variables seleccionadas que mejor explican el fenómeno educativo de las poblaciones indígenas en estudio.

En la especificación el modelo se ha considerado principalmente la definición de Educación Virtual como una forma de educación que puede llevarse en modalidad presencial y/o no presencial. Y justamente promovemos la combinación de ambas (b-learning) considerando el perfil de los grupos participantes indígenas adultos que tienen muy poco conocimiento y acceso a las Tecnologías de información y comunicación, y que requieren de la presencialidad para captar y familiarizarse mejor con ciertos elementos tecnológicos y pedagógicos. Se ha estudiado la propuesta de diferentes modelos de calidad educativa tanto en un enfoque global (de sistemas de evaluación de calidad estándar) como

$14^{\text {th }}$ LACCEI International Multi-Conference for Engineering, Education, and Technology: "Engineering Innovations for Global Sustainability", 20-22 July 2016, San José, Costa Rica. 
en un enfoque parcial con la consideración de distintas dimensiones tales como la dimensión de plataforma tecnológica orientada a evaluar la plataforma como componente virtual, dimensión de evaluación de contenido, dimensión de requisitos, etc. Asimismo, el modelo propuesto y validado, se sustenta en un modelo educativo virtual complementario a la educación regular, que orienta su actividad hacia aprendizajes de corte transversal (educación alternativa permanente); y en analogía al modelo ayllu (forma de organización y modelo social indígena), considera la participación de los pueblos a través de sus representaciones sociales. El modelo validado está constituido por doce áreas o criterios como se indica en la figura 1 .

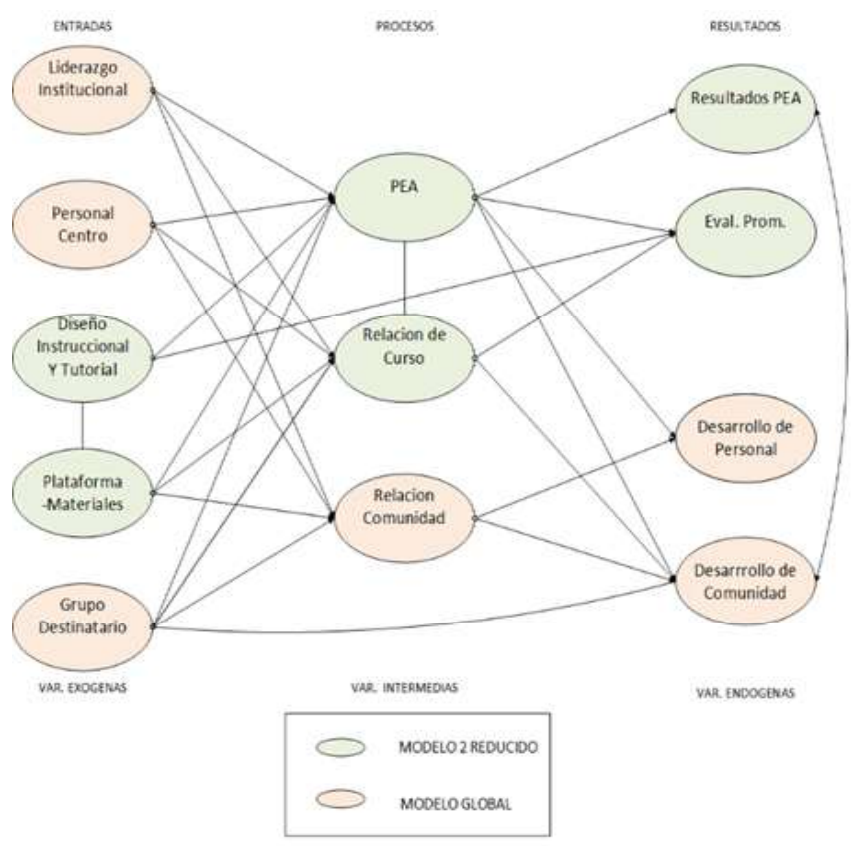

Figura $\mathrm{N}^{\circ} 1$ Modelo validado de Garantía de Calidad en Educación Virtual para Pueblos Indígenas [2].

Se ha agrupado las áreas o criterios de calidad en tres partes principales: Entrada, Proceso y Resultado. La agrupación se orienta a separar por una parte los criterios que se refieren a organización y planificación de cursos virtuales, diseño y elaboración de material, mecanismos de concertación con los pueblos indígenas, organización de los grupos participantes, a lo que denominamos ENTRADA que indica que deben existir o estar de manera previa a la capacitación. Por otra parte, agrupamos los criterios y variables que marcan las relaciones que se producen en el proceso educativo llamado PROCESO DE TRABAJO CLAVE, este elemento es esencial para la evaluación del desempeño organizacional, los procesos de trabajo clave describen cómo se realiza el trabajo en una organización de capacitación, son las tareas y actividades que transforman entradas en salidas tal que la organización puede lograr su propósito educativo. De esta manera, la tercera parte le corresponde a la agrupación de RESULTADOS obtenidos, donde distinguimos los resultados de la capacitación en nuestros participantes destinatarios y hacia donde se enfoca el proceso educativo organizado (resultado de Proceso Enseñanza Aprendizaje PEA), asimismo consideramos el resultado obtenido con el personal de apoyo docente y técnico, quienes participan directamente en el proceso educativo y deben sentirse parte de este en todo momento promoviendo en ellos la actualización y realimentación en el modelo para la mejora continua. La representación corresponde a un modelo causal donde a las variables de entrada denominamos variables causales ya que a partir de ellas se produce el proceso educativo (con Variables Intermedias), el cual, si ha sido debidamente organizado y ejecutado tendrá Resultados satisfactorios, es decir, se habrá logrado los objetivos propuestos para la capacitación.

\subsection{Definición de Criterios}

A continuación se define brevemente cada uno de los elementos del modelo, en base a documentos oficiales del Estado Boliviano y otra literatura consultada. Debemos considerar que, en la aplicación del modelo participan varias instituciones unas de carácter académico que se abocaran a organizar la capacitación; otras de carácter social que representan a los pueblos indígenas y son quienes dan a conocer los requerimientos, cumplen la función de convocatoria y coordinación; y por otro lado las instituciones de carácter político o fiscal, como Alcaldía, corregidor, autoridades educativas, etc. que proveen apoyo logístico, cuestiones de infraestructura y normativa.

\section{Criterio 1: Liderazgo Institucional}

Estudiar cómo se desarrolla y se aplica la estructura organizacional del programa o curso, el marco de los procesos y su sistema de gestión, necesario para la eficaz ejecución de la Política y la Estrategia de las Instituciones participantes, mediante adecuados comportamientos y acciones de los responsables del programa o curso, actuando como líderes.

\section{Criterio 2: Personal Centro}

Analizar cómo la organización del Programa o curso promueve la selección, participación, la formación y el desarrollo del personal del Centro Educativo, tanto personal docente como apoyo administrativo y técnico, de forma individual o equipo, con el fin de contribuir a la eficaz y eficiente gestión del Programa.

\section{Criterio 3: Diseño Instruccional y Tutorial}

Analizar y preparar las bases y fundamentos curriculares atendiendo las demandas de la población objetivo, para lograr aprendizajes significativos, así como la planificación tutorial. Estudio de la demanda de población para un diseño efectivo, Planificar y Preparar guías de curso.

Criterio 4: Plataforma-Materiales 
Analizar y preparar perfil tecnológico, garantizar disponibilidad de equipos y programas informáticos. Revisar naturaleza de equipos, capacidad de acceso y conectividad. Asimismo las bases y fundamentos curriculares atendiendo las demandas de la población objetivo, para lograr aprendizajes significativos. Se refiere a la infraestructura tecnológica (hardware y software); Recursos materiales y alianzas, es decir, al perfil tecnológico, los equipos, material elaborado con los contenidos e información del Programa o curso, programas informáticos disponibles, la naturaleza de los equipos, su capacidad de acceso y la velocidad de conexión a Internet. De igual manera analizar cómo los responsables del programa o curso garantizan el acceso, tienen, mantienen y optimizan los recursos internos y externos, así como la búsqueda de alianzas $\mathrm{y} / \mathrm{o}$ convenios entre instituciones educativas con los municipios y representaciones sociales, para desarrollar la planificación estratégica del programa o curso y la consecución de sus objetivos.

\section{Criterio 5: Grupo destinatario}

Analizar cómo las instituciones organizadoras identifican a los destinatarios y los procesos educativos para el desarrollo del programa, cómo pone en práctica los procesos, cómo los revisa y evalúa para asegurar la mejora del programa.

Criterio 6: Resultados procesos educativos e interacción

Tomar conocimiento y analizar lo que está consiguiendo el programa o curso en relación a sus destinatarios y procesos educativos, grado de satisfacción y resultados de desempeño y rendimiento, obtenidos con la participación en el desarrollo del mismo.

\section{Criterio 7: Evaluación - Promoción}

Analizar lo que está consiguiendo el programa o curso en cuanto a resultados académicos y a satisfacer las necesidades y expectativas de las Instituciones participantes, en relación a rendimiento de los estudiantes. Tener y aplicar modalidades de promoción y certificación.

\section{Criterio 8: Resultados de desarrollo del personal}

Analizar lo que está consiguiendo el programa o curso en relación con el desarrollo del personal involucrado y la existencia de los medios necesarios para el adecuado desempeño de sus funciones. Este criterio es complementario con el criterio 2, referido a apoyo de desarrollo al personal involucrado.

\section{Criterio 9: Resultados globales y de comunidad}

Analizar lo que está consiguiendo el programa o curso (resultados académicos, de gestión o social, a corto, medio y largo plazo, que contribuyan al éxito del mismo) en relación con la ejecución de la planificación estratégica del programa o curso y su repercusión en la satisfacción de las necesidades y expectativas de los agentes implicados en su desarrollo, de las Instituciones participantes, y del entorno local o comunidad, o el ámbito nacional (según proceda). Evaluar la información recibida por parte del personal del programa o curso sobre la percepción de satisfacción de la comunidad.

Criterio 10: Procesos educativos enseñanza aprendizaje PEA

Analizar cómo las instituciones organizadoras identifican a los destinatarios y los procesos educativos enseñanza aprendizaje PEA, para el desarrollo del programa, cómo pone en práctica los procesos e interacción, cómo los revisa y evalúa para asegurar la mejora del programa.

\section{Criterio 11: Relación con la comunidad}

Informar y coordinar a través del relacionamiento con la comunidad sobre la ejecución de la planificación estratégica del programa o curso y su repercusión en la satisfacción de las necesidades y expectativas de los agentes implicados en su desarrollo. Evaluar la información recibida por parte de la comunidad a través del personal del programa en relación con la comunidad.

\section{Criterio 12: Interrelaciones de curso}

Contemplar las relaciones que se establecen durante el proceso de enseñanza aprendizaje PEA, entre los alumnos y entre alumno - profesor, tanto en forma virtual como presencial.

Los criterios indicados constituyen variables de tipo latente o constructos, medibles a través de variables observables o indicadores. Todas estas variables, están respaldadas ampliamente por el artículo 69 incisos 3 y 4 de la Ley de Educación 070 del Estado Plurinacional de Bolivia, que se basa en el modelo Ayllu de organización indígena y los principios de participación, horizontalidad, equidad, complementariedad, y transparencia que reza el artículo 73 de la misma Ley [7].

\subsection{Los Constructos y sus Indicadores}

Las variables que forman parte del modelo, considerando el enfoque de la metodología LISREL, son las siguientes.

A. Constructos exógenos:

$\xi 1$ - Liderazgo Institucional

$\xi 2-$ Personal Centro

$\xi 3$ - Diseño Instruccional y Tutorial

$\xi 4$ - Plataforma - Material

$\xi 5$ - Grupo destinatario

B. Constructos endógenos:

$\eta 1$ - Resultados PEA

$\eta 2$ - Evaluación - Promoción

$\eta 3$ - Desarrollo de personal

$\eta 4$ - Desarrollo de comunidad

$\eta 5$ - Proceso Enseñanza Aprendizaje PEA

$\eta 6$ - Relación comunidad

$\eta 7$ - Relaciones de curso (alumnos, maestros)

C. Variables de garantía de calidad en educación virtual:

14 ${ }^{\text {th }}$ LACCEI International Multi-Conference for Engineering, Education, and Technology: "Engineering Innovations for Global Sustainability", 20-22 July 2016, San José, Costa Rica. 


\begin{tabular}{|c|c|c|}
\hline NUM. & $\begin{array}{l}\text { VAR. DE } \\
\text { MEDIDA }\end{array}$ & $\begin{array}{l}\text { ESPECIFICACIÓN DEL MODELO: LOS CONSTRUCTOS } \\
\text { Y SUS INDICADORES }\end{array}$ \\
\hline & EXOGENAS & $\begin{array}{l}\text { I. LIDERAZGO INSTITUCIONAL } \\
\text { (EQUIPO INSTITUCIONAL MEDICION LIKERT) }\end{array}$ \\
\hline 1 & $\mathrm{X} 1 \mathrm{1.V}$ & $\begin{array}{l}\text { Los enunciados de la actividad planteada al estudiante son } \\
\text { adecuados y fáciles de entender. }\end{array}$ \\
\hline 2 & $\begin{array}{ll}\mathrm{X} 2 & 2 . \mathrm{V} \\
\mathrm{l}\end{array}$ & $\begin{array}{l}\text { Los objetivos de las actividades de aprendizaje del curso } \\
\text { describen resultados que son medibles. }\end{array}$ \\
\hline 3 & $\begin{array}{ll}\mathrm{X} 3 & 3 . \mathrm{V}\end{array}$ & $\begin{array}{l}\text { Los objetivos de aprendizaje involucran habilidades de } \\
\text { pensamiento criticas y habilidades de aprendizaje base } \\
\text { (contenido). }\end{array}$ \\
\hline 4 & $\mathrm{X} 4 \quad 4 . \mathrm{V}$ & $\begin{array}{l}\text { Los objetivos de aprendizaje del curso son indicados claramente } \\
\text { y comprensibles al estudiante. }\end{array}$ \\
\hline 5 & $\begin{array}{ll}\mathrm{X} 5 & 5 . \mathrm{V}\end{array}$ & $\begin{array}{l}\text { Se incorpora una política de evaluación sistemática para la } \\
\text { mejora del curso. }\end{array}$ \\
\hline 6 & $\begin{array}{ll}\mathrm{X} 6 & 6 . \mathrm{V}\end{array}$ & $\begin{array}{l}\text { Los objetivos de aprendizaje del curso se articulan y se } \\
\text { especifican en documentos guía }\end{array}$ \\
\hline 7 & $\mathrm{X} 7 \mathrm{7.V}$ & $\begin{array}{l}\text { El contenido responde a las necesidades e intereses del } \\
\text { estudiantado. }\end{array}$ \\
\hline 8 & $\begin{array}{ll}\mathrm{X} 8 & 8 . \mathrm{V}\end{array}$ & $\begin{array}{l}\text { Se diseñan los objetivos teniendo en cuenta el perfil del } \\
\text { estudiante y las competencias adecuadas a la población. }\end{array}$ \\
\hline 9 & X9 $9 . \mathrm{V}$ & El lenguaje utilizado es adecuado a la población. \\
\hline 10 & $\mathrm{X} 10 \quad 10 . \mathrm{V}$ & $\begin{array}{l}\text { Se adecuan los objetivos del programa con temas transversales } \\
\text { que requiere la población. }\end{array}$ \\
\hline 11 & X11 $11 . \mathrm{V}$ & La organización y temario del curso son fáciles de entender. \\
\hline 12 & $\mathrm{X} 12 \quad 12 . \mathrm{V}$ & $\begin{array}{l}\text { La introducción conduce al estudiante a revisar contenidos sin } \\
\text { dificultad. }\end{array}$ \\
\hline 13 & $\begin{array}{ll}\mathrm{X} 13 & 13 . \mathrm{V}\end{array}$ & $\begin{array}{l}\text { Las expectativas del estudiante con respecto a discusiones y a la } \\
\text { comunicación se indican claramente. }\end{array}$ \\
\hline 14 & $\begin{array}{ll}\mathrm{X} 14 & 14 . \mathrm{V}\end{array}$ & $\begin{array}{l}\text { Los tipos de aseguramiento seleccionados y los métodos usados } \\
\text { para someter aseguramiento son apropiados para el ambiente de } \\
\text { aprender de distancia. }\end{array}$ \\
\hline \multirow[t]{2}{*}{15} & $\begin{array}{ll}\mathrm{X} 15 & 15 . \mathrm{V}\end{array}$ & Se garantiza el acceso continuo de los estudiantes a la plataforma \\
\hline & & $\begin{array}{llll}\text { II. PERSONAL CENTRO } & & \\
\text { (MEDICION COORDINADOR } & \text { DE } & \text { EQUIPO } \\
\text { INSTITUCIONAL) } & & \\
\end{array}$ \\
\hline 16 & $\begin{array}{ll}\mathrm{X} 16 & 16 . \mathrm{V}\end{array}$ & $\begin{array}{l}\text { El/los docentes y/o tutores cuenta(n) con formación académica en } \\
\text { el área de conocimiento del programa o curso }\end{array}$ \\
\hline \multirow[t]{2}{*}{17} & $\begin{array}{ll}\mathrm{X} 17 & 17 . \mathrm{V}\end{array}$ & $\begin{array}{l}\text { El/los docentes y/o tutores tienen conocimiento y experiencia en } \\
\text { educación virtual }\end{array}$ \\
\hline & EXOGENAS & $\begin{array}{l}\text { III. PLATAFORMA - MATERIAL } \\
\text { (EQUIPO INSTITUCIONAL MEDICION LIKERT) }\end{array}$ \\
\hline 18 & $\begin{array}{ll}\mathrm{X} 18 & 18 . \mathrm{V}\end{array}$ & $\begin{array}{l}\text { Los materiales educativos apoyan los objetivos de aprendizaje } \\
\text { indicados y tienen suficiente amplitud y profundidad para que el } \\
\text { estudiante aprenda el tema. }\end{array}$ \\
\hline 19 & $\begin{array}{ll}\mathrm{X} 19 & 19 . \mathrm{V}\end{array}$ & $\begin{array}{l}\text { Los materiales educativos se presentan en formatos apropiados, } \\
\text { en plataforma educativa y son fácilmente accesibles y usables por } \\
\text { el estudiante. }\end{array}$ \\
\hline 20 & $\begin{array}{ll}\mathrm{X} 20 & 20 . \mathrm{V}\end{array}$ & $\begin{array}{l}\text { El propósito de los elementos del curso se muestra claramente } \\
\text { (contenido, métodos instruccionales, tecnologías y materiales de } \\
\text { curso). }\end{array}$ \\
\hline 21 & $\mathrm{X} 21 \quad 21 . \mathrm{V}$ & $\begin{array}{l}\text { Los materiales educativos incluyendo materiales de apoyo, tales } \\
\text { como manuales, videos, CD, y software de computadora son } \\
\text { coherentes con el curso. }\end{array}$ \\
\hline 22 & $\begin{array}{ll}\mathrm{X} 22 & 22 . \mathrm{V}\end{array}$ & $\begin{array}{l}\text { Todos los recursos y materiales usados en el curso en línea, están } \\
\text { citados apropiadamente. }\end{array}$ \\
\hline 23 & $\begin{array}{ll}\mathrm{X} 23 & 23 . \mathrm{V}\end{array}$ & $\begin{array}{l}\text { Las herramientas y los medios apoyan los objetivos de } \\
\text { aprendizaje del curso y se relacionan e integran con los textos y } \\
\text { actividades asignadas. }\end{array}$ \\
\hline 24 & $\begin{array}{ll}\mathrm{X} 24 & 24 . \mathrm{V}\end{array}$ & $\begin{array}{l}\text { Las tecnologías requeridas para este curso son fácilmente } \\
\text { descargables. }\end{array}$ \\
\hline 25 & $\begin{array}{ll}\mathrm{X} 25 & 25 . \mathrm{V}\end{array}$ & $\begin{array}{l}\text { Las herramientas y los medios son compatibles con estándares } \\
\text { existentes de los modos de difusión. }\end{array}$ \\
\hline 26 & $\begin{array}{ll}\mathrm{X} 26 & 26 . \mathrm{V}\end{array}$ & $\begin{array}{l}\text { Las instrucciones de acceso a recursos a distancia son suficientes } \\
\text { y fáciles de entender. }\end{array}$ \\
\hline 27 & $\begin{array}{ll}\mathrm{X} 27 & 27 . \mathrm{V}\end{array}$ & $\begin{array}{l}\text { Los recursos adicionales proporcionan alternativas equivalentes } \\
\text { al contenido auditivo y visual. }\end{array}$ \\
\hline 28 & $\begin{array}{ll}\mathrm{X} 28 & 28 . \mathrm{V}\end{array}$ & $\begin{array}{l}\text { Los recursos tienen enlaces que son auto-descriptivos y son } \\
\text { significativos }\end{array}$ \\
\hline 29 & $\begin{array}{ll}X 29 & 29 . V\end{array}$ & $\begin{array}{l}\text { El curso reconoce la importancia de los requisitos de norma } \\
\text { SCORM u otras y los aplica (reusabilidad, adaptabilidad..) }\end{array}$ \\
\hline
\end{tabular}

\begin{tabular}{|c|c|c|}
\hline 30 & $\begin{array}{ll}\mathrm{X} 30 & 30 . \mathrm{V}\end{array}$ & $\begin{array}{l}\text { Se Recoge, almacena y usa información sobre investigación } \\
\text { educativa para la mejora continua del programa. }\end{array}$ \\
\hline 31 & $31 . \mathrm{V}$ & $\begin{array}{l}\text { Se busca equipos, materiales y tecnologías innovadoras para la } \\
\text { mejora continua del curso. }\end{array}$ \\
\hline 32 & $\mathrm{X} 32 \quad 32 . \mathrm{V}$ & $\begin{array}{l}\text { Mantiene en buen estado los equipos, ambientes de trabajo, } \\
\text { materiales y tecnología para mejorar el rendimiento total durante } \\
\text { el desarrollo del curso. }\end{array}$ \\
\hline 33 & $\mathrm{X} 3333 . \mathrm{V}$ & $\begin{array}{l}\text { Se dispone o elabora un reservorio de contenidos que permitan la } \\
\text { sostenibilidad de la capacitación rural }\end{array}$ \\
\hline 34 & X34 $34 . \mathrm{V}$ & $\begin{array}{l}\text { Se establece un sistema de entrada y salida de información } \\
\text { relacionado con el curso. }\end{array}$ \\
\hline 35 & $\begin{array}{l}\mathrm{X} 44 \quad 44 . \mathrm{V} \\
\end{array}$ & $\begin{array}{l}\text { Las actividades de aprendizaje promueven el logro de objetivos } \\
\text { indicados y de resultados de aprendizaje. }\end{array}$ \\
\hline 36 & X45 $45 . \mathrm{V}$ & $\begin{array}{l}\text { Los estándares claros se fijan para la respuesta y la disponibilidad } \\
\text { (el tiempo del instructor alrededor del e-mail, etc.) }\end{array}$ \\
\hline 37 & X46 $46 . \mathrm{V}$ & $\begin{array}{l}\text { El diseño del curso incita al tutor a estar presente, activo y } \\
\text { enganchado con los estudiantes. }\end{array}$ \\
\hline 38 & $\mathrm{X} 47 \quad 47 . \mathrm{V}$ & $\begin{array}{l}\text { Las instrucciones de curso articulan o se ligan a una descripción } \\
\text { clara de la ayuda técnica ofrecida. }\end{array}$ \\
\hline 39 & $\begin{array}{ll}\mathrm{X} 48 & 48 . \mathrm{V}\end{array}$ & $\begin{array}{l}\text { Las instrucciones de curso articulan o se ligan a una explicación } \\
\text { de cómo el sistema de ayuda académico de la institución puede } \\
\text { asistir al estudiante a usar con eficiencia los recursos } \\
\text { proporcionados. }\end{array}$ \\
\hline 40 & $\begin{array}{ll}\mathrm{X} 49 & 49 . \mathrm{V}\end{array}$ & $\begin{array}{l}\text { La plataforma educativa utilizada es adecuada al programa, y } \\
\text { provee todas las funcionalidades requeridas }\end{array}$ \\
\hline 41 & $50 . \mathrm{V}$ & $\begin{array}{l}\text { Las instrucciones de curso articulan o acoplan a las clases } \\
\text { impartidas y a recursos que contengan las preguntas básicas } \\
\text { relacionadas con la realidad temática, la escritura, la tecnología, } \\
\text { etc. }\end{array}$ \\
\hline 42 & X51 $51 . \mathrm{V}$ & $\begin{array}{l}\text { La plataforma tiene presentación sencilla, entendible y es fácil } \\
\text { de acceder }\end{array}$ \\
\hline 43 & $52 . \mathrm{V}$ & $\begin{array}{l}\text { Los requisitos mínimos de la tecnología, las habilidades mínimas } \\
\text { del estudiante, el conocimiento necesario en la disciplina, se } \\
\text { indica claramente }\end{array}$ \\
\hline \multirow[t]{2}{*}{44} & $53 . \mathrm{V}$ & $\begin{array}{l}\text { La plataforma educativa utilizada es adecuada al perfil de los } \\
\text { estudiantes de la comunidad, y provee facilidades para el } \\
\text { aprendizaje }\end{array}$ \\
\hline & & (MEDICION LIKERT POR ESTUDIANTES) \\
\hline 45 & $\mathrm{X} 40 \quad 40 . \mathrm{V}$ & $\begin{array}{l}\text { Las herramientas y los medios apoyan los objetivos de } \\
\text { aprendizaje del curso y se relacionan e integran con los textos y } \\
\text { actividades asignadas }\end{array}$ \\
\hline 46 & $41 . \mathrm{V}$ & $\begin{array}{l}\text { Las tecnologías requeridas para este curso son fácilmente } \\
\text { descargables. }\end{array}$ \\
\hline 47 & $\mathrm{X} 42 \quad 42 . \mathrm{V}$ & $\begin{array}{l}\text { Las instrucciones de acceso a recursos a distancia son suficientes } \\
\text { y fáciles de entender. }\end{array}$ \\
\hline 48 & $\begin{array}{ll}\mathrm{X} 43 & 43 . \mathrm{V}\end{array}$ & $\begin{array}{l}\text { Los recursos adicionales y ejemplos apoyan objetivamente el } \\
\text { aprendizaje. }\end{array}$ \\
\hline 49 & $\mathrm{X} 54 \quad 54 . \mathrm{V}$ & $\begin{array}{l}\text { La plataforma tiene presentación sencilla, entendible y es fácil } \\
\text { de acceder }\end{array}$ \\
\hline \multirow[t]{2}{*}{50} & $\begin{array}{ll}\mathrm{X} 55 & 55 . \mathrm{V}\end{array}$ & $\begin{array}{l}\text { Se fijan los estándares claros para la respuesta y la disponibilidad } \\
\text { (el tiempo del instructor alrededor del e-mail, etc.) }\end{array}$ \\
\hline & EXOGENAS & $\begin{array}{l}\text { IV. DISEÑO INSTRUCCIONAL - TUTORIAL } \\
\text { (MEDICION LIKERT POR ESTUDIANTES) }\end{array}$ \\
\hline 51 & $\mathrm{X} 36 \quad 36 . \mathrm{V}$ & $\begin{array}{l}\text { Los materiales educativos apoyan los objetivos de aprendizaje } \\
\text { indicados y tienen suficiente amplitud y profundidad para que el } \\
\text { estudiante aprenda el tema. }\end{array}$ \\
\hline 52 & $\mathrm{X} 38 \quad 38 . \mathrm{V}$ & $\begin{array}{l}\text { El propósito de los elementos del curso se muestra claramente } \\
\text { (contenido, métodos instruccionales, tecnologías y materiales de } \\
\text { curso). }\end{array}$ \\
\hline 53 & $\begin{array}{ll}\mathrm{X} 56 & 56 . \mathrm{V}\end{array}$ & $\begin{array}{l}\text { Los requisitos para la interacción del curso se articulan } \\
\text { claramente. }\end{array}$ \\
\hline \multirow[t]{2}{*}{54} & X57 $57 . \mathrm{V}$ & $\begin{array}{l}\text { El diseño del curso incita al tutor a estar presente, activo y } \\
\text { enganchado con los estudiantes. }\end{array}$ \\
\hline & EXOGENAS & $\begin{array}{l}\text { V. GRUPO DESTINATARIO } \\
\text { (EQUIPO INSTITUCIONAL MEDICION LIKERT) } \\
\end{array}$ \\
\hline 55 & $\begin{array}{ll}\mathrm{X} 58 & 58 . \mathrm{V}\end{array}$ & $\begin{array}{l}\text { La política de aprobación y promoción es transparente y fácil de } \\
\text { entender. }\end{array}$ \\
\hline 56 & $59 . \mathrm{V}$ & $\begin{array}{l}\text { Las estrategias de aseguramiento y medición proveen } \\
\text { realimentación al estudiante. }\end{array}$ \\
\hline 57 & $\begin{array}{ll}\mathrm{X} 60 & 60 . \mathrm{V}\end{array}$ & $\begin{array}{l}\text { Los tipos de aseguramiento seleccionados y los métodos usados } \\
\text { para someter aseguramiento son apropiados para el ambiente de } \\
\text { aprender de distancia. }\end{array}$ \\
\hline 58 & $61 . \mathrm{V}$ & Se garantiza el acceso continuo de los estudiantes a la plataforma \\
\hline
\end{tabular}

$14^{\text {th }}$ LACCEI International Multi-Conference for Engineering, Education, and Technology: "Engineering Innovations for Global Sustainability", 20-22 July 2016, San José, Costa Rica. 


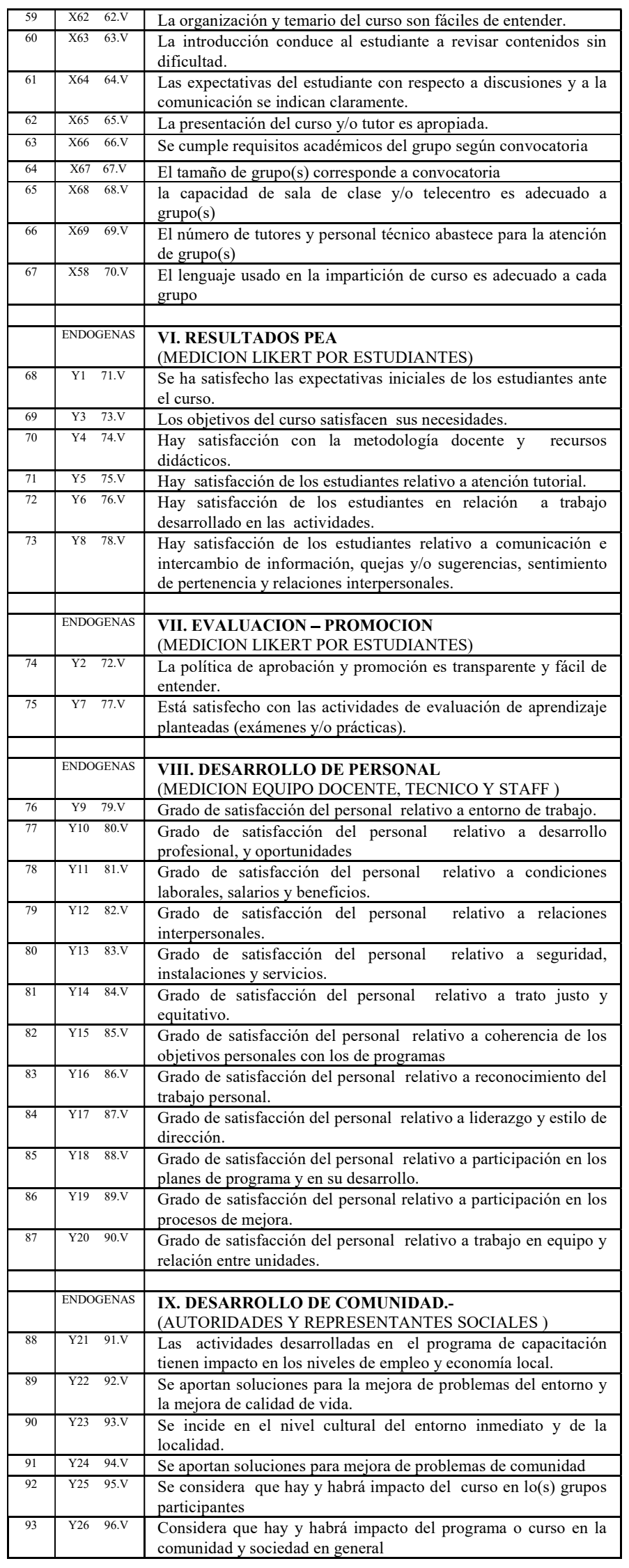

\begin{tabular}{|c|c|c|}
\hline 94 & Y27 97.V & Indique su grado de satisfacción por esta capacitación \\
\hline \multirow[t]{2}{*}{95} & $98 . \mathrm{V}$ & Considera importante continuar con capacitación en TIC'S \\
\hline & INTERMEDIAS & $\begin{array}{l}\text { X PROCESO DE ENSEÑANZA APRENDIZAJE PEA } \\
\text { (EQUIPO INSTITUCIONAL MEDICION LIKERT) }\end{array}$ \\
\hline 96 & $\begin{array}{ll}\text { Y29 } & 99 . \mathrm{V}\end{array}$ & $\begin{array}{l}\text { Los tipos de aseguramiento seleccionados miden los objetivos de } \\
\text { aprendizaje indicados y son coherentes con actividades y recursos } \\
\text { del curso. }\end{array}$ \\
\hline 97 & $\begin{array}{ll}\text { Y30 } & 100 . \mathrm{V}\end{array}$ & $\begin{array}{l}\text { La Política de aprobación y promoción es transparente y fácil de } \\
\text { entender }\end{array}$ \\
\hline \multirow[t]{2}{*}{98} & $101 . \mathrm{V}$ & $\begin{array}{l}\begin{array}{l}\text { Las estrategias de aseguramiento } \\
\text { realimentación al estudiante. }\end{array} \\
\end{array}$ \\
\hline & & (MEDICION LIKERT POR ESTUDIANTES) \\
\hline 99 & $\begin{array}{ll}\text { Y32 } & 102 . \mathrm{V} \\
\end{array}$ & La organización y temario del curso son fáciles de entender. \\
\hline 100 & $103 . \mathrm{V}$ & $\begin{array}{l}\text { Las expectativas del estudiante con respecto a discusiones y a la } \\
\text { comunicación se indican claramente (se dispone de una guía u } \\
\text { otro). }\end{array}$ \\
\hline 101 & $\begin{array}{ll}\text { Y34 } & 104 . V \\
\end{array}$ & La presentación del curso y/o tutor es apropiada. \\
\hline 102 & $\begin{array}{ll}\text { Y35 } & 105 . \mathrm{V}\end{array}$ & $\begin{array}{l}\begin{array}{l}\text { Los enunciados de la actividad planteada al estudiante son } \\
\text { adecuados y fáciles de entender }\end{array} \\
\end{array}$ \\
\hline 103 & $\begin{array}{ll}\text { Y36 } & 106 . \mathrm{V}\end{array}$ & $\begin{array}{l}\text { Los objetivos de aprendizaje del curso son indicados claramente } \\
\text { y comprensibles al estudiante }\end{array}$ \\
\hline \multirow[t]{2}{*}{$\begin{array}{l}10 \\
4\end{array}$} & $109 . \mathrm{V}$ & $\begin{array}{l}\text { Los requisitos para la interacción del curso se articulan } \\
\text { claramente. }\end{array}$ \\
\hline & INTERMEDIAS & $\begin{array}{l}\text { XI RELACION CON LA COMUNIDAD } \\
\text { (MEDICION COORDINADOR EQUIPO INSTITUCIONAL) }\end{array}$ \\
\hline 105 & $107 . \mathrm{V}$ & Se ha suscrito compromiso, acuerdo o convenio interinstitucional \\
\hline \multirow[t]{2}{*}{106} & $108 . \mathrm{V}$ & $\begin{array}{l}\text { Existe coordinación con la comunidad para impartir el programa } \\
\text { o curso }\end{array}$ \\
\hline & INTERMEDIAS & $\begin{array}{l}\text { XII RELACION DE CURSO } \\
\text { (MEDICION LIKERT POR ESTUDIANTES) }\end{array}$ \\
\hline 107 & $\begin{array}{ll}\text { Y40 } & 110 . \mathrm{V}\end{array}$ & $\begin{array}{l}\text { Las actividades de aprendizaje fomentan interacción tutor- } \\
\text { estudiante }\end{array}$ \\
\hline 108 & $111 . \mathrm{V}$ & $\begin{array}{l}\text { Las actividades de aprendizaje fomentan interacción estudiante- } \\
\text { estudiante. }\end{array}$ \\
\hline
\end{tabular}

Cuadro $\mathrm{N}^{\circ}$ 1. Los constructos y sus indicadores (2)

Se tiene en total: 108 variables, siendo 67 variables exógenas y 41 variables endógenas.

A continuación se presenta el modelo estructural que muestra los indicadores del cuadro distribuidos en el modelo de medida de variables exógenas y el modelo de medida de variables endógenas. 


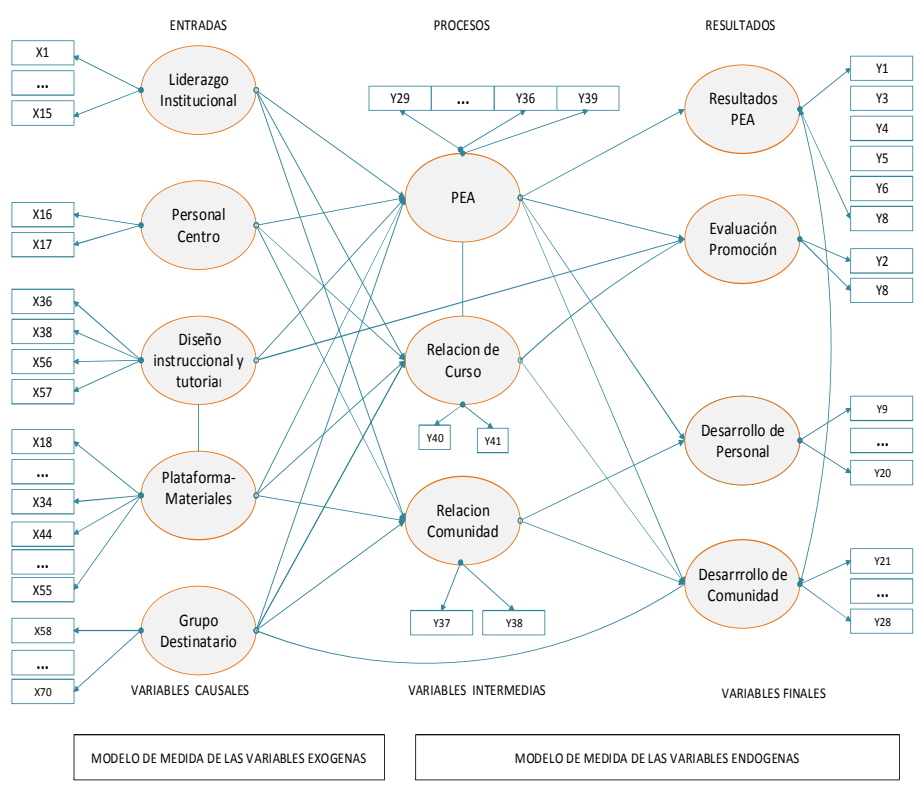

Figura $\mathrm{N}^{\circ} 2$. Modelo Estructural con indicadores de constructos

\subsection{Instrumentos de recogida de datos}

Los instrumentos de recogida de datos elaborados específicamente para el Modelo son cuestionarios de encuesta a aplicarse por tres grupos identificados como agentes de evaluación:

i) El equipo institucional conformado por el personal de la institución académica

ii) Los grupos participantes en la capacitación (estudiantes)

iii) Autoridades de la comunidad y representantes sociales

Se tiene tres cuestionarios, uno para cada grupo identificado como agente evaluador.

i) Cuestionario para el equipo Institucional. El equipo institucional como organizador de la capacitación manejará un formulario inicial donde figuran todos los indicadores correspondientes a organización, el cual permite verificar que en la planificación y preparación del curso se haya considerado todos los elementos, y ajustar si es el caso. Por este motivo este primer cuestionario se aplicará durante la etapa de organización y planificación. Este cuestionario quedó formado por 64 ítems correspondientes a 15 ítems de factor 'Liderazgo Institucional'; 2 ítems de factor 'personal centro'; 27 ítems de factor 'plataforma y material'; 3 ítems de factor 'PEA'; 2 ítems de 'Interrelación alumno maestro'; 13 ítems de factor 'Grupo Destinatario' y 2 ítems de 'Relacióncomunidad'. De manera separada se aplicó también a este grupo institucional el cuestionario correspondiente a factor 'Desarrollo de Personal' con 12 ítems (al finalizar la capacitación). Son en total 76 ítems para el equipo institucional.

ii) Cuestionario para los estudiantes. El cuestionario para llenar por los estudiantes se aplica durante la etapa de capacitación. Este cuestionario quedó formado por 26 ítems correspondientes a 6 ítems de factor 'Plataforma - material'; 4 ítems de factor 'diseño instruccional tutorial'; 6 ítems de factor 'Resultados PEA'; 2 ítems de factor 'Evaluaciónpromoción'; 6 ítems de factor 'PEA'; 3 ítems de 'relación de curso'.

iii) Cuestionario para autoridades y representantes sociales. El cuestionario para autoridades y representaciones sociales, se aplica al finalizar la capacitación. Este cuestionario quedó constituido por 8 ítems correspondientes a factor 'Desarrollo de Comunidad'.

\subsection{Escala de medición}

En cuanto a la formulación de preguntas y respuestas, se aplica la escala tipo Likert con cuatro alternativas $(1,2,3,4)$. Algunos autores consideran que cinco es el número adecuado, otros indican que el número par o impar de respuestas es irrelevante; se utilizará un número par para evitar la tendencia de situarse en el punto medio de la escala [8]. Por tanto, para todos los ítems de los cuestionarios, la escala de respuestas se ha fijado de la siguiente manera:

\begin{tabular}{|c|c|c|c|}
\hline 1 & 2 & 3 & 4 \\
\hline NADA & POCO & BASTANTE & MUCHO \\
\hline $\begin{array}{c}\text { RESPUESTAS VALORADAS } \\
\text { NEGATIVAS }\end{array}$ & $\begin{array}{c}\text { RESPUESTAS VALORADAS } \\
\text { POSITIVAS }\end{array}$ \\
\hline
\end{tabular}

Cuadro $\mathrm{N}^{\circ}$ 2. Escala de Medición tipo Likert [2]

\subsection{Población y muestra}

Debido a las limitaciones de capacidad en los Telecentros Educativos Comunitarios TEC's (tienen 10 a 15 equipos de computación) y la consideración que se ha hecho de tener grupos casi homogéneos, se espera entonces que los grupos participantes sean relativamente pequeños, esto es, 10 a 15 personas por cada grupo. Por ello, se recomienda aplicar el cuestionario de estudiantes a todo el universo del Programa o curso.

2.6 Actividades en capacitación según modelo de educación virtual para pueblos indigenas

Realizar las actividades que se indican a continuación, en el marco de las características del modelo de educación virtual para pueblos indígenas.

$1^{\circ}$ Organización y planificación, revisión de 3 componentes:

- Contenidos de aprendizaje significativo, estudio demanda

- Tecnología, en Población indígena destinataria

- Pedagogía, se recomienda aplicar estrategias (Motivar para iniciar y mantener el interés por aprender, Explicitar los objetivos, Presentar contenidos significativos y funcionales, Incentivar la autoformación, Potenciar el trabajo colaborativo en grupos de aprendizaje, evaluar

$14^{\text {th }}$ LACCEI International Multi-Conference for Engineering, Education, and Technology: "Engineering Innovations for 
progreso, Respetar la diversidad de los grupos, Promover la transferencia de los aprendizajes) y la consideración.

$2^{\circ}$ Diseño instruccional y tutorial, tareas ISD:

- Planificación, identificando y analizando los fundamentos del curso.

- Diseño, estableciendo la estructura y forma en que se presentará los contenidos del curso virtual

- Desarrollo, obteniendo los productos diseñados en formato digital, cargado y pruebas

- Puesta en práctica, utilizando las herramientas y estrategias de enseñanza adecuadas

- Evaluación, determinando el cumplimiento de objetivos.

$3^{\circ}$ Virtualización de contenidos:

Existen varias normas, tales como: IEEE LTSC., IMS, AICC., SCORM, ISO, etc. Pero se trabajó con SCORM y herramienta RELOAD EDITOR.

$4^{\circ}$ Capacitación en Pueblos Indigenes, en sitio y a distancia:

- Se recomienda combinar E.V. presencial con E.V. no presencial (b-learning)

- Conformación de grupos en lo posible homogéneos

- Configurar y publicar la plataforma a utilizar

- Distribuir el material elaborado en plataforma, así como impresos

- Preparar y distribuir la guía de curso con indicaciones básicas de organización, contenidos, objetivos, trabajos prácticos, etc.

- Se recomienda entregar la guía de curso con las debidas explicaciones en una sesión o reunión presencial

$5^{\circ}$ Garantía de calidad:

En todo el desarrollo del programa aplicar las encuestas para asegurar la calidad del proceso educativo las cuales son propias del modelo de garantía de calidad en educación virtual para poblaciones indígenas de Bolivia construido y aplicado para tal fin.

\subsection{Análisis de variables y su medición}

Durante el desarrollo de las capacitaciones manejar los datos obtenidos de las encuestas organizados por criterios de calidad y calculando las percentiles por criterio para tener la información rápida y realizar los ajustes a los estándares indicados por el modelo, obteniendo en su momento los cuadros de datos y gráficas por criterio. A continuación se muestra un cuadro de procesamiento por criterio de calidad calculando \%. Si por cada criterio se obtiene $\%>70$ se está logrando los objetivos de calidad, sino revisar causas, analizar y ajustar (este uso es de manera opcional, se puede optimizar generando gráficas y aumentar la exigencia de calidad por ejemplo $\%>80$ ).

\begin{tabular}{|c|c|c|c|c|}
\hline CRIIERIOS & \begin{tabular}{|c|} 
MAXIMO \\
PUNTAJE (MP)
\end{tabular} & $\begin{array}{l}\text { PUNTOS } \\
\text { CRITERIO }\end{array}$ & $\%$ & OBS. \\
\hline I.LIDERAZG0 (15ITEMS) & $(15 * 4)$ & SUMA-PUNTOS I & SUNA-PUNTOS I*100MP & \multirow{12}{*}{$\begin{array}{l}\stackrel{0}{i} \\
\stackrel{0}{0}\end{array}$} \\
\hline I. PERSONAL CENTRO (2 ITEMS) & $(2 * 4)$ & SUMA-PUNTOS II & SUAAA-PUNTOS $\Pi^{*} 100 / A P$ & \\
\hline III. PLATAFORMA- MATERLAL (33 ITEMS) & $(33 * 4)$ & SUNA-PUNTOS III & SUMA-PUNTOS II*100/NP & \\
\hline N. DISENO INSTRUCCIONAL - TUTORLAL (4 ITEMS) & $(4 * 4)$ & SUNA-PUNTOS N $\mathrm{N}$ & SUMA-PUNTOS IV*100/NP & \\
\hline V. GRUPOS DESTINATARIOS (13 ITEMS) & $(13 * 4)$ & SUMA-PUNTOS V & SLMA-PUNTOS V*100/MP & \\
\hline V. RESULTADOS PEA (6 6 TEMS) & $(6 * 4)$ & SUNA-PUNTOS VI & SUMA-PUNTOS VI*100/MP & \\
\hline VII. EVALUACION-PROMOCION (2 ITEMS) & $(2 * 4)$ & SUNA-PUNTOS VII & SUMA-PUNTOS VI"100/NP & \\
\hline VIII. DESARROLLO DE PERSONAL (12 ITEMS) & $(12 * 4)$ & SUMA-PTNTOS VIII & $\begin{array}{l}\text { SUMA-PUNTOS } \\
\text { VIII'100AP }\end{array}$ & \\
\hline IX DESARROLLO DE COMUNIDAD (8 ITEMS) & $(8 * 4)$ & SUNA-PUNTOS IX & SUMA-PUNTOS LX*100MP & \\
\hline $\begin{array}{l}\text { X PROCESO DE ENSEX̀ANZA APRENDIZAJE PEA }(9 \\
\text { ITEMS) }\end{array}$ & $(9 \div 4)$ & SUMA-PUNTOS $\mathrm{X}$ & SLMA-PUNTOS $X^{*} 100 / \mathrm{NP}$ & \\
\hline XI RELACION CON LA COMUNIDAD (2 IENS) & $(2 * 4)$ & SUNA-PUNTOS XI & SCNA-PUNTOS XI*100/MP & \\
\hline $\begin{array}{l}\text { III RELACION DE CURSO ALUNNO MAESTRO (2 } \\
\text { ITEMS) }\end{array}$ & $(2 * 4)$ & SUNAA-PUNTOS XII & SUMA-PUNTOS XII $100 / \mathrm{MP}$ & \\
\hline
\end{tabular}

Cuadro $N^{\circ} 3$. Datos de Procesamiento de evaluación por criterio

\section{INTERPRETACIÓN DEL MODELO RE-ESPECIFICADO}

En cuanto a interpretación del modelo y relación entre las variables latentes, se ha encontrado influencias directas e indirectas en el modelo re-especificado en cuestión. De esta manera, quedaron demostradas las siguientes relaciones causales que se presentaron como hipótesis del trabajo de investigación [2]:

1. Existe un efecto directo entre el 'PEA' y las actividades de 'Liderazgo institucional'

2. Existe un efecto directo entre 'Relación De Curso' y 'Liderazgo institucional'

3. Existe un efecto directo entre 'Relación Comunidad' y 'Liderazgo institucional'

4. Existe un efecto directo entre el 'PEA' y el 'Personal del Centro'

5. Existe un efecto directo entre el 'Relación De Curso' y el 'Personal del Centro'

6. Existe un efecto directo entre el 'Relación Comunidad' y el 'Personal del Centro'

7. Existe un efecto directo entre 'PEA' y 'Diseño Instruccional Tutorial'

8. Existe un efecto directo entre 'Evaluación - Promoción' $\mathrm{y}$ 'Diseño Instruccional Tutorial'

9. Existe un efecto directo entre 'PEA' y 'Plataforma Materiales'

10. Existe un efecto directo entre 'Relación de Curso' y 'Plataforma - Materiales'

11. Existe un efecto directo entre 'Relación Comunidad' y 'Plataforma - Materiales'

12. Existe un efecto directo entre 'PEA' y 'grupo destinatario'

13. Existe un efecto directo entre 'Relación de Curso' y 'grupo destinatario'

14. Existe un efecto directo entre 'Relación Comunidad' y 'grupo destinatario'

15. Existe un efecto directo entre 'Desarrollo de Comunidad' y 'grupo destinatario'

16. Existe un efecto directo entre 'Resultados PEA' y 'PEA'

$14^{\text {th }}$ LACCEI International Multi-Conference for Engineering, Education, and Technology: "Engineering Innovations for Global Sustainability", 20-22 July 2016, San José, Costa Rica. 
17. Existe un efecto directo entre 'Resultados PEA' y 'Desarrollo de Comunidad'

18. Existe un efecto directo entre 'Evaluación - Promoción' y 'Relación de Curso'

19. Existe un efecto directo entre 'Desarrollo de Comunidad' y 'Relación de Curso'

20. Existe un efecto directo entre 'Desarrollo de Personal' y 'PEA'

21. Existe un efecto directo entre 'Desarrollo de Comunidad' $y$ 'PEA'

22. Existe un efecto directo entre 'Evaluación - Promoción' y 'PEA'

23. Existe un efecto directo entre 'Desarrollo de Comunidad' y 'Relación de Curso'

24. Existe un efecto directo entre 'Desarrollo de Comunidad' $\mathrm{y}$ 'Relación Comunidad'

25. Existe un efecto directo entre 'Desarrollo de Personal' y 'Relación Comunidad'

El modelo presentado ha sido suficientemente validado en dos proyectos de capacitación semi-presencial en Pueblos Indígenas de la Amazonía boliviana siguiendo todos los pasos establecidos indicados. Con los datos obtenidos empíricamente se ha realizado el análisis factorial dentro de la metodología causal aplicada para la estimación de parámetros y validación del modelo.

\section{AgRADECIMIENTO}

Nuestro agradecimiento a quienes han apoyado la realización de este trabajo: a las autoridades de los cantones de San Buenaventura y Tumupasa, al DIPGIS UMSA por otorgar parte del financiamiento requerido, y a quienes participaron en el equipo institucional durante las capacitaciones, así como al gobierno regional de Madrid (eMadrid S2013/ICE-2715)

\section{REFERENCIAS}

[1] INE. BOLIVIA. Caracteristicas de Poblacion y vivienda. La Paz: INE, 2012.

[2] DOLZ, Fátima C., Tesis Doctoral. Garantia de calidad en Educacion Virtual No Formal para Pueblos Indígenas. Madrid : Archivo UPM, 2016.

[3] SAAVEDRA, Bautista. EL AYLLU. Estudios sociológicos. La Paz, Bolivia : Juventud, 1971.

[4] Hernandez Pina, Fuensanta. Los modelos causales aplicados al estudio de las variables de aprendizaje de una L2. 1990, Universidad de Murcia.

[5] CASAS, Mercedes. Los Modelos de Ecuaciones Estructurales y su Aplicacion en el Indice Europeo de Satisfaccion del Cliente. Universitat Valencia. [En línea] 08 de 2009. http://www.uv.es/asepuma/X/C29C.pdf.

[6] ALDAS MANZANO, J. Analisis Factorial Confirmatorio. [ed.] hompson. Analisis Multvariante Aplicado . Madrid : Paraninfo, 2005, pág. 351.

[7] Estado Plurinacional de Bolivia, Ministerio de Educacion y Cultura. Ley de Educacion Avelino Siñani - Elizardo Perez $N^{\circ}$ 70. La Paz: Asamblea Constituyente, 2010.

[8] Ruiz de Miguel, C. Propuesta y validacion de un modelo de calidad en Educacion Infantil. Madrid : Universidad Complutense de Madrid, 2002.
BIBLIOGRAFÍA

ABET. (Abril de 2007). Leadership and Quality assurance in Applied Sciencem, computing, Engineering, and Technology Education.Obtenido de ABET: http://www.abet.org

Accino, J. A. (2006). AGORA VIRTUAL: Una propuesta de entorno colaborativo y de enseñanza sobre interfaces OSID. Boletin RedIris $N^{\circ}$ 76.

Asamblea Constituyente. Republica de Bolivia. Nueva Constitucion Politica del Estado. Sucre, Boliva : Version oficial, 2008, pág. 54.

ADL. (2006). Sharable Content Object Reference Model. Advanced Distributed Learning. Obtenido de ADL: www.ADLNet.gov

Colace et al. (2006). Evaluation Models for e-learning Platform: an AHP approach, in Frontiers in Education Conference,. 36th Annual 2006, IEEE.

Correa, J. (2000). "Calidad Educativa On line? Analisis de la Calidad de la Educacion Universitaria basada en Internet.Obtenido de www.say.us.es

Costabile et al. (2005). "On the Usability Evaluation of E-Learning Applications". 38th Hawai International Conference on System Sciences. Hawai: IEEE.

De la Fuente Fernandez, S. (2013). Numeros Indices. Obtenido de Universidad Autonoma de Madrid: http://www.fuenterrebollo.com/Economicas2013/indices-teoria.pdf

DETC. (Abril de 2007). The Distance Education and Training Council.Obtenido de DETC: http://www.detc.org

ECA. (marzo de 2010). European Consortium for Accreditation in higher education.Obtenido de ECA web site: http://www.eurashe.eu/

ECONOTICIAS, B. (12 de julio de 2006). La Educacion Boliviana sufre una gran frustracion. Obtenido de EconoticiasBolivia.com: http://www.econoticiasbolivia.com/documentos/notadeldia/congreedu5.ht $\mathrm{m}$

EFQM. (2007). Modelo de excelencia. Obtenido de EFQM: http://www.efqm.org/

ENQA. (marzo de 2007). European Association for Quality Assurance in Higher Education.Obtenido de ENQA: http://www.enqa.eu

Garcia Aretio, L. (2006). La Educacion a Distancia. De la Teoria a la Practica. Barcelona: Ariel S.A.

Garcia Veiga, M. A. (2011). Analisis Causal con ecuacones Estructurales de la Satisfaccion ciudadana con los servicios municipales. Santiago de Compostela: Universidad Santiago de Compostela.

GATE-UPM, G. d.-e. (2005). Determinacion de un modelo causal de los factores de calidad docente en entornos virtuales de aprendizaje. Obtenido de UPM: www.mec.es/univ/proyectos2005/EA2005-0146.pdf

Gefen and Ridings. (2005). "A Sociolinguistics Perspectiva of gender Differences in Virtual Communities". The Data Base for Advances in Information Systems, pág 78-92.

Grutzner et al. (2004). "Improving Courseware Quality through Life-Cycle Encompassing Quality Assurance". Symposium on Applied Computing. Nicosia: Cyprus ACM Press.

Gulliver and Ghinea. (2006). "Defining User Perception of Distributed Multimedia Quality". ACM Transactions on Multimedia Computing, communications and Applications, (págs. 241-257).

INQAAHE. (mayo de 2007). International Network for Quality Assurance Agencies in Higher Education.Obtenido de INQAAHE: http://www.inqaahe.org/

Jaramillo, L. (2015). Blended learning y la educacion a distancia. VI Congreso Virtual Iberoamericano de Calidad en Educacion Virtual y a distancia.Educ@2015.

Kaczmarczyk, L. (2001). "Accreditation and Student Assessment in Distance Education: Why We Need to Pay Attention". Annual Joint Conference Integrating Technology into computer Science Education . Canterbury: United Kingdom ACM Press.

Loayza Melgarejo, M. (2006). La evaluacion de la calidad en la Educacion Virtual. Analisis y Propuesta. Sucre, Bolivia: Talleres gráficos UASB.

Lopez Feal, R. Los modelos causales asociados a estructuras de Covarianza: Una via para la Unificacion delos "dos metodos de la Psicologia Cientifica". Barcelona: Departamento de Psicologia Expermental.

$14^{\text {th }}$ LACCEI International Multi-Conference for Engineering, Education, and Technology: "Engineering Innovations for 
Marshall and Mitchell. (2004). "Applying SPICE to e-learning: An e-learning Maturity Model". Sixth Australasian Computing Education Conference ACE2004. Dunedin, Nueva Zelanda.

MEC, M. d. (octubre de 2006). TECNOLOGÍAS DE LA INFORMACIÓN Y COMUNICACIÓN. Obtenido de PROGRAMA NACIONAL DE TICs: http://www.gesci.org/old/files/docman/About_Programa_Nacional_de_TI CS.pdf

Miguel A. Ruiz, A. P. (2010). MODELOS DE ECUACIONES ESTRUCTURALES. Papeles del Psicólogo, pp. 34-45 http://www.cop.es/, Vol. 31(1) ( Universidad Autónoma de Madrid), 110.

MILLER, B. (2007). Assessng Organitacional Performance in Higher Education. En B. MILLER, Assessng Organitacional Performance in Higher Education.San Francisco. USA.

MIN. EDUCACION Y CULTURAS, M. (2006). "Aprendiendo en la Diversidad ". Obtenido de Educabolivia: www.educabolivia.bo

Sandoval, C. (2014). Estandares de Calidad en el e-learning. V Congreso Internacional sobre Calidad y Accesibilidad de la Formación Virtual (CAFVIR 2014). Guatemala: Universidad Galileo.

Sangra, A. (2002). La calidad en las experiencias virtuales de Educacion superior.Barcelona.
3) Preparar el Programa en base a objetivos y requerimiento de las poblaciones, realizar el diseño Curricular enunciando en cada contenido los objetivos (Diseño instruccional y Tutorial).

4) Organizar las actividades a desarrollar en el PEA en coordinación con la comunidad ('Liderazgo' e 'Interacción con la Comunidad).

5) Si no se dispone del personal capacitado para las tareas específicas de capacitación en Pueblos Indígenas convocar públicamente a personal con el perfil necesario para dictar cursos virtuales en TIC's ('Personal de Centro').

6) Elaborar guías del contenido curricular y el material de apoyo didáctico, así como los objetos de aprendizaje.

7) Preparación y configuración de las plataformas educativas analizando sus características y funcionalidades adecuadas a características de la población destinataria.

8) Desarrollar coordinación permanente y apoyo mutuo entre Organizaciones de Convenio.

9) Establecer cronograma de trabajo y horarios de atención o reunión, y convocatoria a inscripción en consenso con las representaciones sociales, autoridades y población indígena y a través de ellos.

10) Evaluar los resultados y rendimiento de PEA, preparando y entregando la certificación a quienes fueron realizando las actividades educativas y trabajos prácticos solicitados en jornadas de capacitación.

11) Medir los parámetros observables del modelo propuesto (ítems de liderazgo, personal, ítems de proceso, de resultados de proceso, y resultados de Desarrollo) aplicando encuestas a cada agente evaluador durante las etapas indicadas.

12) Organizar los Objetos de Aprendizaje en un Repositorio de Objetos para ser utilizados por otras comunidades

ANEXO

GUIA DE USO DEL MODELO DE GARANTÍA DE CALIDAD EN EDUCACIÓN VIRTUAL PARA PUEBLOS INDÍGENAS

El modelo de garantía de calidad en Educación Virtual para pueblos indígenas es aplicable en procesos educativos de capacitación o formación alternativa continua, en modalidad virtual presencial y no presencial (blearning).

1) Se debe tener o establecer de manera previa un convenio marco entre la institución Académica, el Consejo Indígena $\mathrm{u}$ otro como Representación Social, la Alcaldía Municipal, y la Sub-alcaldía local, para generar y desarrollar proyectos de investigación y programas de Capacitación que promuevan el desarrollo de la Región (factores 'Recursos, Materiales y Alianzas' y 'Desarrollo de Comunidad').

2) Se debe hacer un estudio de la demanda de capacitación formación. Esto a fin de generar contenidos de aprendizaje significativo que es la base de nuestro Modelo de Educación Virtual para Pueblos Indígenas. 